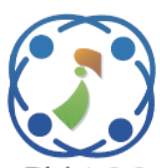

\title{
Coordinated Optimal Placement of Energy Storage System and Capacitor Bank Considering Optimal Energy Storage Scheduling for Distribution System Using Mixed-Integer Particle Swarm Optimization
}

\author{
Korawitch Kaiyawong $^{1} \quad$ Keerati Chayakulkheeree $^{1 *}$ \\ ${ }^{1}$ School of Electrical Engineering, Institute of Engineering, Suranaree University of Technology, \\ Nakhonratchasima, Thailand \\ * Corresponding author's Email: keerati.ch@sut.ac.th
}

\begin{abstract}
This paper proposes a mixed-integer particle swarm optimization (MIPSO) for coordinated optimal placement of energy storage system (ESS) and capacitor bank (CB). In the propose method, optimal ESS scheduling (OESSS) is solved by particle swarm optimization (PSO), as a subproblem, the optimal coordinated placement (COP) for ESS and CB, simultaneously. The distribution system annual loss minimization (DSALM) is used as the objectives of COP problem. The proposed method was tested with the IEEE 33-bus radial distribution test system. The results demonstrated that the proposed method is successful and robust in minimizing system losses, which loss saving of $35.12 \%$ when compare to the based case, which is the best solution among other existing methods.
\end{abstract}

Keywords: Optimal placement, Optimal scheduling, Minimize power loss, Energy storage system, Capacitor bank.

\section{Introduction}

Nowadays, in distribution system, day-to-day fluctuations in power consumption. distributed high, resulting in significant power loss in the system. Therefore, a reduction in power loss and improved voltage are very important for the electrical distribution power systems (EDPs) to reduce energy consumption and operating costs. This problem can be solved by installing capacitor bank (CB) to compensate for reactive power and power consumption variations that can be managed with an energy storage system (ESS).

A number of methods have been presented with the purpose of reducing power loss and improving voltage in electrical power systems. such as, distribution system reconfiguration using modified particle swarm optimization (MPSO) [1], Soft Open Point (SOP) which is a type of power electrical component which can be used to replace traditional switches or normally open points (NOP) in network distribution was proposed in [2]. In [3], parameter improved particle swarm optimization (PIPSO) is solver for optimal sizing and placement of a distributed generation (DG), this paper integrating real power supporting of DG with IEEE 33 bus and 69 bus radial distribution network. CB has been used to solve optimal dispatch problem for reactive power in [4]. Particle swarm optimization (PSO) was utilized to test a power system to reduction in a power loss in [5]. In other system of this challenge, [6] calculated the operation of $\mathrm{CB}$ and tap-changing transformer using the Newton-Raphson load flow technique. When minimizing power loss is integrated with other aims, such as controlling voltage drop in the electrical system, fuzzy multi-objective optimization has been used genetic algorithms (GA) for control device in power system [7]. These methods, aimed at minimizing real power loss.

Optimal placement of CB is a method of locating the proper $\mathrm{CB}$ installation for the electrical system's best benefit. The capacitor placement challenge is a very well-known topic that has been discussed by a number of authors in the past, such as, genetic algorithm method $[8,9]$, the sperm whale algorithm (SWA) [10], moth-flame optimization algorithm [11] and complex calculation methods like fuzzy logic 
control (FLC) [12], these methods are used for reduce power loss, improve the voltage profile but not considering operation cost. In addition to other considerations, the cost of installing and operating capacitors will be considered, such as cuckoo search algorithm (CSA) [13], locust search method (LS) [14], particle swarm optimization (PSO) [15, 16], genetic algorithm (GA) [17], and hybrid approach of PSO-GA [18]. All the above methods can efficiently reduce the operating cost of the installed capacitor bank in the distribution system. [19] determining the size and location of reactive power compensation integrated with hydro power distributed generation into the power system. In the presence of distributed solar power generation (PV), [20] develop a model of a specific distribution system to select the appropriate capacity and location sets of newly installed CB, these prove that capacitors can be used in conjunction with EDPs for minimize power loss.

Similar to other resources, ESS can be solved in a multitude of situations, including optimizing capacity, increasing stability, balancing supply fluctuations, satisfying a load demand. However, under or over-voltage in the distribution system can be caused by inappropriate ESS allocation and sizing. Therefore, optimal placement of ESS (OPESS) is important for planning location and sizing of ESS, to maximize the benefits from ESS. Many research's used OPESS for integrating renewable energy sources (RER). For examples, [21] propose a wind generator with ESS to improve voltage profile and system loss using the PSO algorithm for optimal placement in the 34-bus unbalanced system. Some researches focus on integrating ESS with power PV station, such as, the optimal siting of battery energy storage system (BESS) in distribution network is solved by PSO to minimize the energy losses of the system [22, 23]. With similar objective function, [2426] developed the GA optimization method to reduce daily loss and peak demand by PV stations while deploying ESS, but the cost of installing ESS is not considered. With the same concept, [27] developed a GA optimization approach for minimizing voltage fluctuations induced by PV penetration by distributing BESS across permitted nodes of a distribution system while considering for capital, land-of-use, and installation costs with a qualitative cost model. Therefore, OPESS can efficiently locate and install ESS to benefit the system.

In addition to finding the proper installation location for ESS, optimal scheduling of energy storage systems is also important for day-to-day operation. [28] presented a model based on LaGrange relaxation and tests with the IEEE 37 bus considering reshaping the load curve when using ESS. [29] used the exchange market algorithm (EMA), when ESS is installed with RER uncertainty. The 24-hour optimal scheduling for ESS using load forecasting and RER is developed in [30, 31] with prediction of the load forecast and operation time of PV in the short term. All of the above method confirms that ESS can manage energy allocation at various periods of the day to reduce power loss or peak demand load. However, the break-even point of today's ESS deployments isn't worth contemplating. Thereby, the high cost of ESS poses severe concerns, necessitating the development of cost-effective alternatives [32]. In addition, the optimal placement of ESS can be gain more benefit when incorporation the problem with CB placement.

Most of researches on optimal CB placement solve the total loss minimization using single loading condition. Meanwhile, the optimal scheduling of ESS is solved without optimal allocation. Therefore, this paper proposed the method for solving the optimal placement of $\mathrm{CB}$ and ESS, simultaneously, considering optimal scheduling of ESS.

In this paper, coordinated optimal placement problem (COPP) of ESS and CB considering optimal ESS scheduling (OESSS) for distribution system annual loss minimization (DSALM) is proposed. The proposed method used the mixed-integer particle swarm optimization (MIPSO) to optimal placement of ESS and CBs, while OESSS is solved by PSO [33]. Thailand's power system load profile was used to verify the proposed method in the radial IEEE 33-bus distribution test system. The results shown that the proposed method can successfully provide optimal ESS and CB allocation.

In this paper, the COPP formulation is addressed in Section 2. Section 3 presents a MIPSO approach to the COPP. Section 4 shows the results of the radial distribution IEEE-33 bus test system. Lastly, Section 5 address the conclusion.

\section{Problem formulation}

The proposed method includes coordinated optimal placement problem (COPP) formulations considering daily ESS scheduling using MIPSO algorithm. The structure of a distribution grid typically includes active and reactive resources such as, bulk power systems, ESS, CB, and load demand, as shown in Fig. 1.

The objective function of COPP is formulated as follows:

Minimize

$$
A A L=D P_{\text {loss, total }}\left(\boldsymbol{C}_{\text {ess }}^{\boldsymbol{h}}, \boldsymbol{C B S}, \boldsymbol{B}\right),
$$




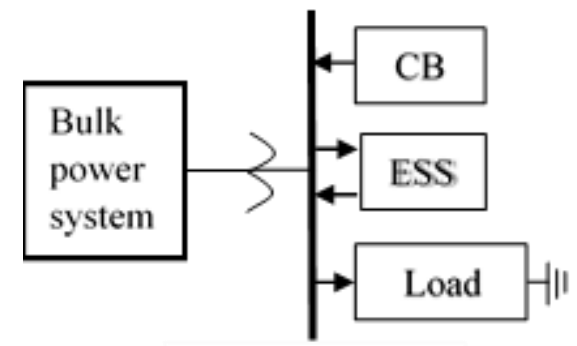

Distribution bus

Figure. 1 A structure of distribution system with $\mathrm{CB}$ and ESS

for ESS and CB connected to bus $\mathbf{B}$ with state of charge $\mathbf{C}^{\mathbf{h}}$ ess and compensated from $\mathbf{C B S}$.

The considered time interval is one day ( 24 hour),

$$
P_{\text {loss }, \text { total }}=\sum_{h=1}^{24} P_{\text {loss }}^{h}
$$

where,

$$
\begin{gathered}
P_{l o s s}^{h}=\sum_{i=1}^{N B} \sum_{\substack{j=1 \\
j \neq i}}^{N B} G_{i j}\left[\left(V_{i}^{h}\right)^{2}+\left(V_{j}^{h}\right)^{2}-\right. \\
\left.2 V_{i}^{h} V_{j}^{h} \cos \left(\delta_{i}^{h}-\delta_{j}^{h}\right)\right] \\
\text { for } i, j=1, \ldots, N B, h=1, \ldots, 24
\end{gathered}
$$

Subject to the power balance constraints,

$$
\begin{gathered}
P_{G i}^{h}-P_{D i}^{h}+\left(C_{e s s, i}^{h} \times\left(\frac{\eta_{c}}{\eta_{d}}\right)\right)= \\
\sum_{j=1}^{N B}\left|V_{i}^{h}\right|\left|V_{j}^{h}\right|\left|y_{i j}\right| \cos \left(\theta_{i j}-\delta_{i j}^{h}\right) \\
\quad \text { for } i=1, \ldots, N B, h=1, \ldots, 24 \\
Q_{G i}^{h}-Q_{D i}^{h}-c b s_{i}=-\sum_{j=1}^{N B}\left|V_{i}^{h}\right|\left|V_{j}^{h}\right|\left|y_{i j}\right| \sin \left(\theta_{i j}-\right. \\
\left.\delta_{i j}^{h}\right) \text {,for } i=1, \ldots, N B, h=1, \ldots, 24
\end{gathered}
$$

Line flow limit constraints,

$$
\left|f_{i}^{h}\right| \leq f_{i}^{\max }, \text { for } i=1, \ldots, N L, h=1, \ldots, 24,(6)
$$

Power generation constraint,

$$
\begin{gathered}
P_{G i}^{\min } \leq P_{G i}^{h} \leq P_{G i}^{\max }, \\
\text { for } i=1, \ldots, N G, h=1, \ldots, 24, \\
\quad Q_{G i}^{\min } \leq Q_{G i}^{h} \leq Q_{G i}^{\max }, \\
\text { for } i=1, \ldots, N G, h=1, \ldots, 24,
\end{gathered}
$$

Bus voltage limit constraint,

$$
\begin{aligned}
& \left|V_{i}^{\min }\right| \leq\left|V_{i}^{h}\right| \leq\left|V_{i}^{\max }\right| \\
& \text { for } i=1, \ldots, N B, h=1, \ldots, 24
\end{aligned}
$$

The reactive power generation of $\mathrm{CB}$ can be represented as,

$$
\begin{gathered}
\boldsymbol{C B S}=\left[c b s_{1}, c b s_{2}, \ldots, c b s_{i}, \ldots, c b s_{N C B}\right] \\
\text { for } i=1, \ldots, N C B
\end{gathered}
$$

The energy capacity, as well as the capacity for power charging and discharging, are all included in this paper's ESS model. [31] shows the efficiency of charging and discharging represented by [34],

$$
\begin{aligned}
& \boldsymbol{E S}=\left[E S_{i}^{1}, \ldots, E S_{i}^{h}, \ldots E S_{i}^{24}\right], \\
& \text { for } i=1, \ldots, N E S S, h=1, \ldots, 24 \text {, } \\
& 0 \leq E S_{i}^{h} \leq E S_{i}^{h, \max } \\
& \text { for } i=1, \ldots, N E S S, h=1, \ldots, 24 \text {, } \\
& \boldsymbol{C}_{\text {ess }}=\left[C_{e s s, i}^{1}, \ldots, C_{e s s, i}^{h}, \ldots C_{e s s, i}^{24}\right] \\
& \text { for } i=1, \ldots, N E S S, h=1, \ldots, 24 \text {, } \\
& C_{e s s, i}^{h, \min } \leq C_{e s s, i}^{h} \leq C_{e s s, i}^{h, \max } \\
& \text { for } i=1, \ldots, N E S S, h=1, \ldots, 24 \text {, } \\
& C_{e s s, i}^{h}=\left\{\begin{array}{l}
E S_{i}^{1}, h=1 \\
E S_{i}^{h}-E S_{i}^{h-1}, h=2, \ldots, 24
\end{array}\right. \\
& \text { for } i=1, \ldots, N E S S, h=1, \ldots, 24 \text {. }
\end{aligned}
$$

In this paper, if $C_{e s s, i}^{h}<0$, the ESS is in discharging condition, if $C_{\text {ess }, i}^{h}>0$, the ESS is in charging condition.

The initial set of particles for bus that connected with ESS and CB as,

$$
\begin{gathered}
\boldsymbol{B}=\left[b_{1}, \ldots, b_{i}, \ldots, b_{N E S S+N C B}\right]^{T}, \\
\text { for } 1 \leq b_{i} \leq N B, \\
b_{i} \in\{\text { integer }\} \\
i=1, \ldots,(N E S S+N C B) .
\end{gathered}
$$

where,

$A A L \quad$ is average annual loss $(\mathrm{kWh})$,

$D \quad$ is number of days,

$P_{\text {loss,total }}$ is the total daily loss,

$P_{\text {loss }}^{h}$ is the hourly loss in each hour,

$G_{i j} \quad$ is the conductance of the lines between bus $i$ and bus $j$ for $j \neq i$,

$V_{i j}{ }^{h} \quad$ is the voltage of bus $i, j$ in each hour,

$\delta_{i j}{ }^{h} \quad$ is the voltage angle difference between bus $i$ and $j$ in each hour,

$P_{G i}^{h} \quad$ is active power of generator connected bus $i$ in each hour,

$P_{G i}{ }^{\min }$ is minimize active power generation,

$P_{G i}^{\max }$ is maximum active power generation, 
$Q_{G i}{ }^{h}$ is reactive power of generator connected bus $i$ in each hour,

$Q_{G i}{ }^{m i n}$ is minimize reactive power generation,

$Q_{G i}{ }^{\max }$ is maximum reactive power generation,

$f_{i} \quad$ is the MVA flow of line $i$ in each hour is $f_{i}{ }^{h}$,

$f_{i}^{\max }$ is the limit of line flow (MVA),

$\left|V_{i}\right|^{h} \quad$ is the voltage magnitude of bus $i$,

$V_{i}^{\text {min }}$ is minimum voltage magnitude for bus $i$,

$\left|V_{i}\right|^{\max }$ is maximum voltage magnitude for bus $i$,

$\left|y_{i j}\right| \quad$ is the magnitude of the $y_{i j}$ element of $Y_{b u s}$.,

$\theta_{i j} \quad$ is the angle of the $y_{i j}$ element of $Y_{b u s}$,

$N B$ is the total number of buses,

$N G$ is the total number of generators,

$N L$ is the total number of lines,

CBS is the matrix representing size of $\mathrm{CB}$,

$c b s_{i} \quad$ is the size of CB to be installed,

$N C B$ is the total number of CB in the system,

ES is the matrix representing capacity of ESS,

$E S_{i}^{h} \quad$ is the capacity of $i^{\text {th }}$ ESS at hour $h$,

$\mathbf{C}_{\text {ess }}$ is the matrix of charge/discharge by ESS,

$C^{h}{ }_{e s s, i}$ is charge/discharge of $i^{\text {th }} \mathrm{ESS}$ at hour $h$,

$\eta_{c}, \eta_{d}$ are the charging and discharging efficiency,

NESS is the total number of ESS in the system,

B is the matrix representing bus number with ESS and CB,

$b_{i} \quad$ is the bus number connected ESS and CB.

\section{MIPSO based COPP algorithm}

In this paper, the set of particles for bus number connected with ESS (B) in Eq. (16) was rounded to identify the location of ESS and CBs using Eq. (17). Subsequently, initial set of particles for schedule is obtained by Eqs. (11) to (15).

The schedule of $\mathbf{C}^{\mathbf{h}}$ ess in Eq. (15) is obtained by PSO. $\mathbf{C}^{\mathbf{h}}$ ess and CBS in Eq. (10) are used for obtain $A A L$ in Eq. (1), the scheduling and placement were update position by PSO [33]. The annual loss is utilized as the objective function in Eq. (1), which is based on the load flow analysis. The minimum value of $A A L$ among all particles is called gbest, and the minimum $A A L$ of individual ith particle is called pbest. The COPP computational procedure as shown is Fig. 2.

\section{Result and discussion}

The proposed MIRPSO based COPP has been verified on the IEEE 33-bus radial distribution test system shown in Fig. 3. The system line data and bus data were obtained from [14].

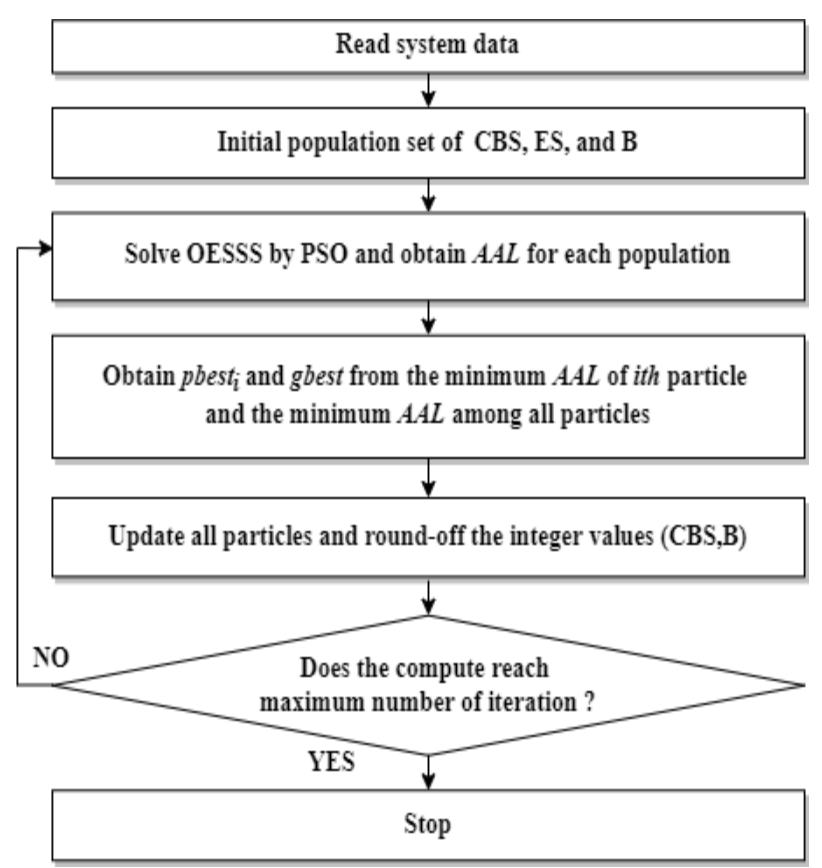

Figure. 2 The COPP computational procedure

The Thailand daily load curve on 14 April 2018, shown in Fig. 4. which is annual peak day, is used as the system load profile.

The simulation study includes,

Case 1: Reference case (without ESS and CBs),

Case 2: Optimal placement of ESS,

Case 3: Optimal placement of CBs,

Case 4: COPP of ESS and CBs.

\subsection{Reference case (without ESS and CBs)}

In this case, the IEEE 33-bus radial distribution test system without energy storage system and capacitor bank was solved by Newton-Raphson power flow for obtain $A A L$. The result illustrates the system average annual loss without capacitor bank and energy storage system is $129.78 \mathrm{kWh}$. The result $A A L$ in this case used to compare with other cases.

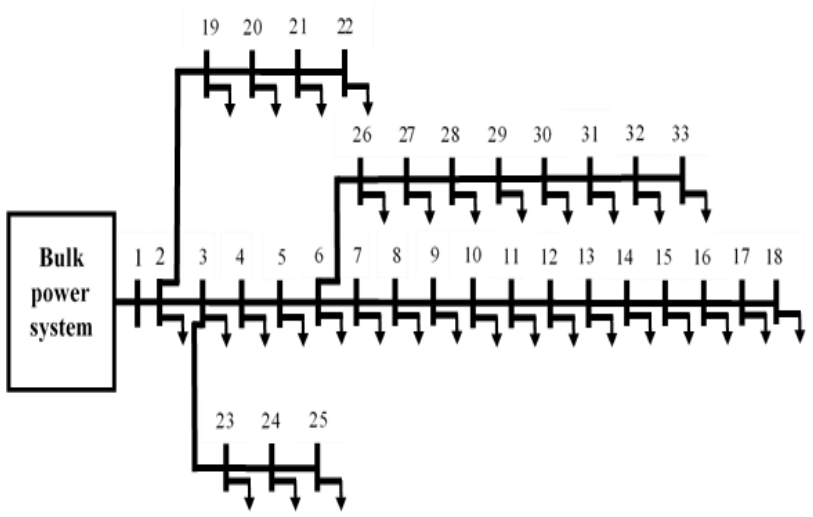

Figure. 3 IEEE 33-bus radial distribution test system 


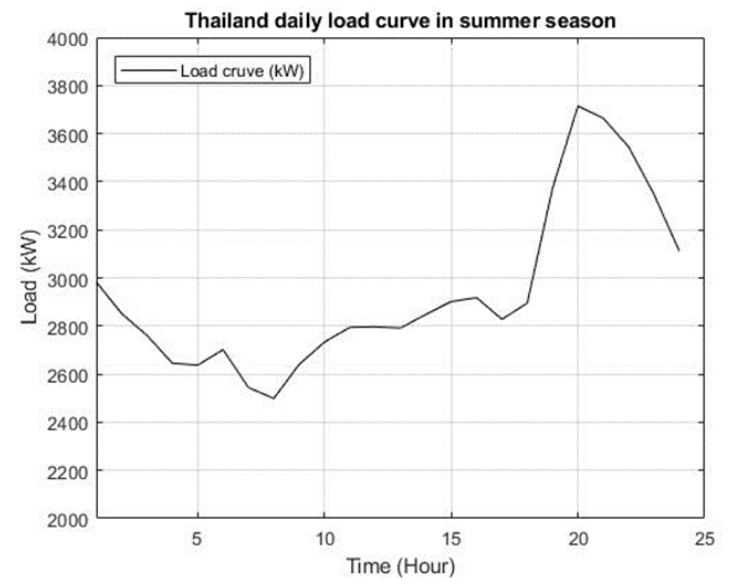

Figure. 4 Thailand daily load curve for test system

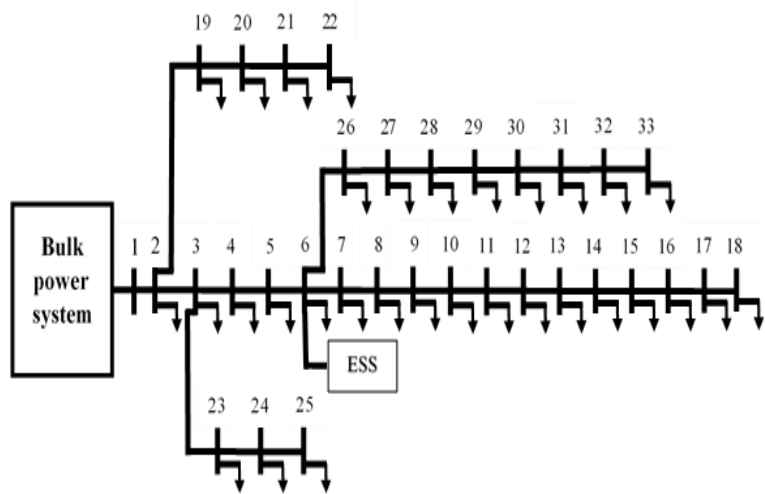

Figure. 5 The modified IEEE 33-bus radial distribution test system with ESS

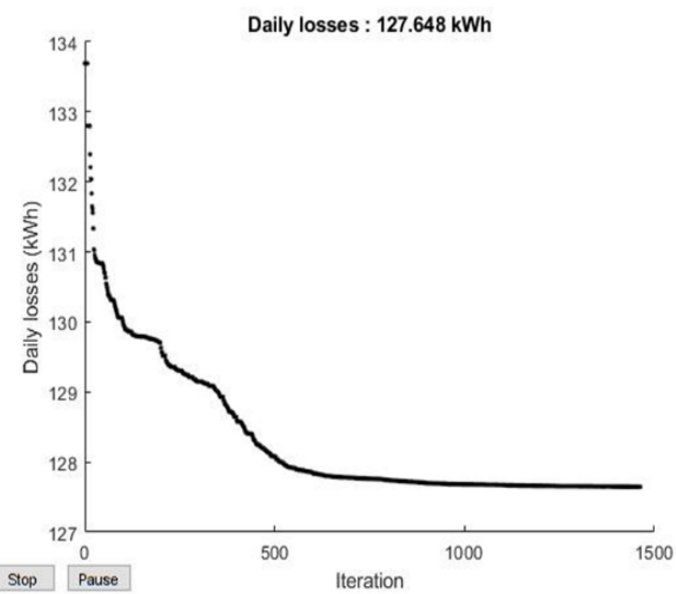

Figure. 6 Daily loss in each iteration

\subsection{Optimal placement of ESS}

In this case, the size $1.5 \mathrm{MW} / 10 \mathrm{MWh}$ ESS is used to test the propose algorithm. The optimal ESS was investigated by the proposed MIPSO considering OESSS. As shown in Fig. 5, bus number 6 was chosen for install the ESS. In this case, the $A A L$ with OPESS is $127.65 \mathrm{kWh}$, Fig. 6 shows the convergence plot of solution.

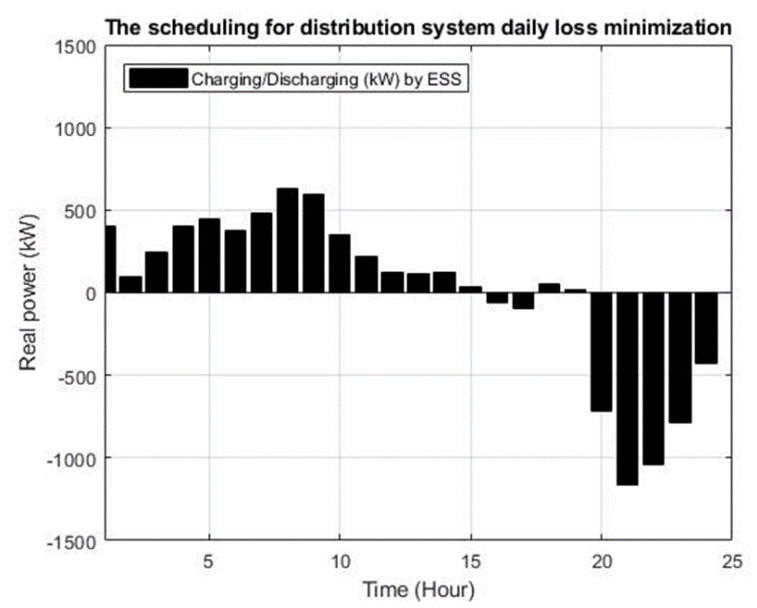

Figure. 7 The scheduling for distribution system daily loss minimization

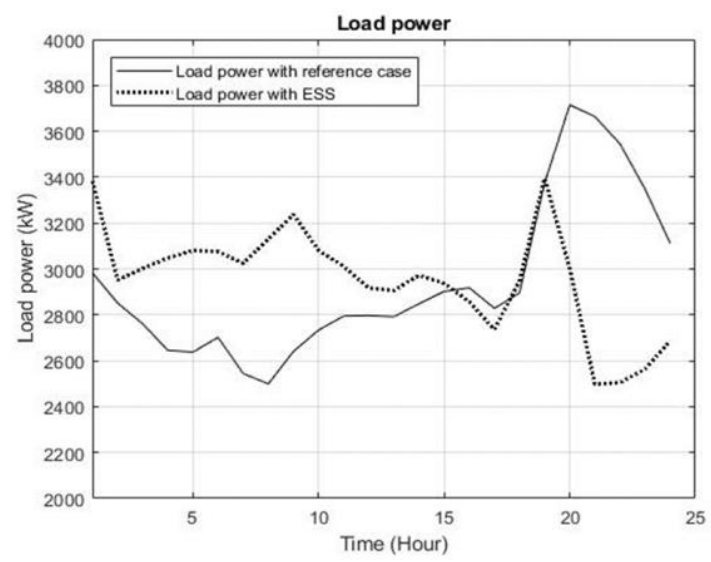

Figure. 8 The comparison of power load with and without ESS

The scheduling of ESS is shown in Fig. 7. Due to ESS is charging when off-peak and discharging when peak load, so peak power load with ESS was decease at 21.00 o'clock, as shown in Fig. 8. ESS is possible to handle high peak demand loads. However, it is still significantly unable to compensate for the system loss and voltage decrease. As a result, CB can assist in the solution of this problem.

\subsection{Optimal placement of CBs}

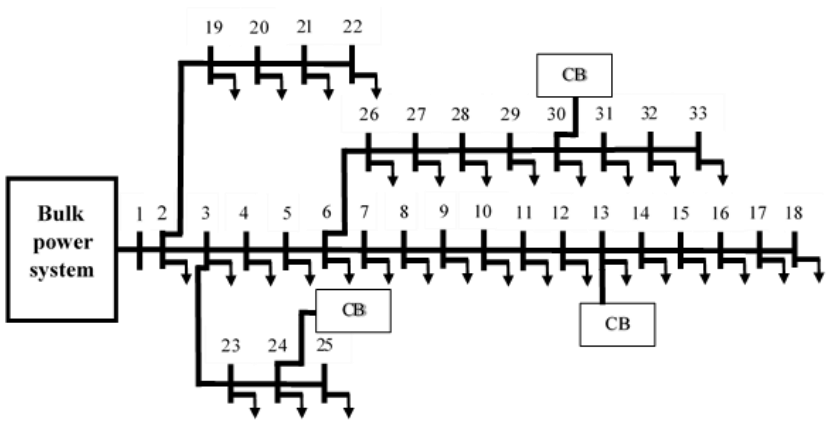

Figure. 9 The modified IEEE 33-bus radial distribution test system with optimal placement $\mathrm{CBs}$ 
Table 1. Comparison method to minimize total loss in optimal CB placement

\begin{tabular}{|c|c|c|c|c|}
\hline Solver & - & LS [14] & \multicolumn{2}{c|}{ MIPSO } \\
\hline Case & Ref. & $\begin{array}{c}\text { Without } \\
\text { DLP }\end{array}$ & $\begin{array}{c}\text { Without } \\
\text { DLP }\end{array}$ & $\begin{array}{c}\text { With } \\
\text { DLP }\end{array}$ \\
\hline Total Loss(kWh) & 210.98 & 139.23 & 139.23 & 86.01 \\
\hline MaximumV(p.u.) & 1.0000 & 1.0000 & 1.0000 & 1.0000 \\
\hline MinimumV(p.u.) & 0.9038 & 0.9291 & 0.9291 & 0.9423 \\
\hline Loss Saving(\%) & $\mathbf{0 . 0 0}$ & $\mathbf{3 4 . 0 1}$ & $\mathbf{3 4 . 0 1}$ & $\mathbf{5 9 . 2 3}$ \\
\hline
\end{tabular}

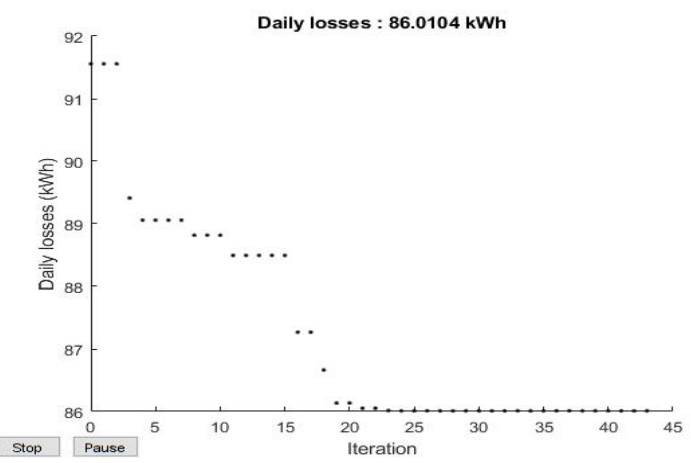

Figure. 10 Daily loss in each iteration

The optimal CB placement in the IEEE 33-bus radial distribution test system by the proposed method was compared to other previous published research, with the aim of minimizing total loss and operation cost [14]. Table 1 shows that the proposed method resulted in the same solution with [14] for conventional problem formulation. However, the daily load profile (DLP) was not considered in [14].

Therefore, in this paper, the DLP was considered to solve for the $A A L$. The 350,600 , and $1050 \mathrm{kVar}$ $\mathrm{CBs}$ are used for optimal placement. From the propose method, CB was installed at bus 13,24 and 30, as shown in Fig. 9. The $A A L$ of this case is $86.0104 \mathrm{kWh}$, which loss saving of $59.23 \%$ (saving more than without DLP case). Fig. 10 shows that the algorithm convergence to minimum loss in 43 iterations. The voltage profile in each bus was compensated condition by MIPSO. The voltage drop in this case is lower than in the reference case and ESS case, as shown in Fig. 16.

\subsection{COPP of ESS and CBs}

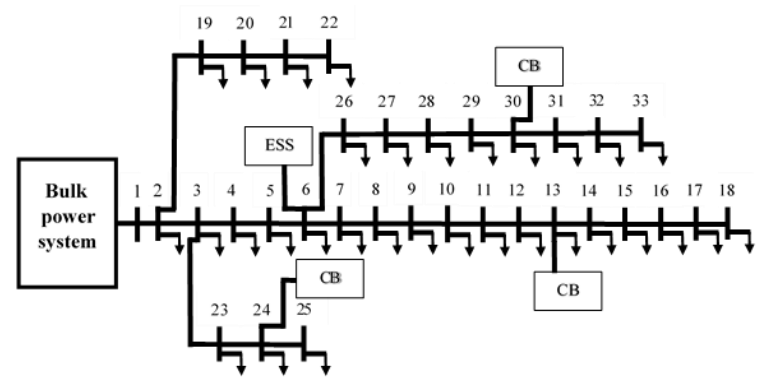

Figure. 11 The modified IEEE 33-bus radial distribution test system with optimal placement ESS and CBs

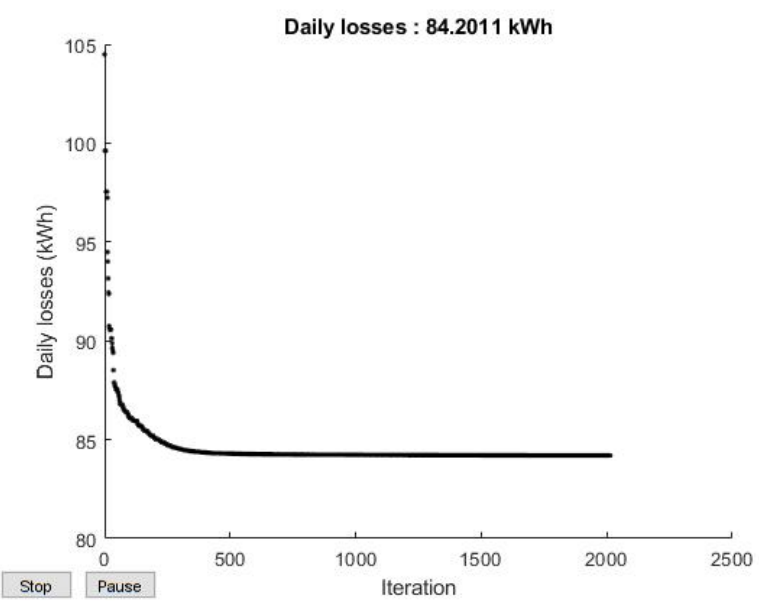

Figure. 12 Daily loss in each iteration

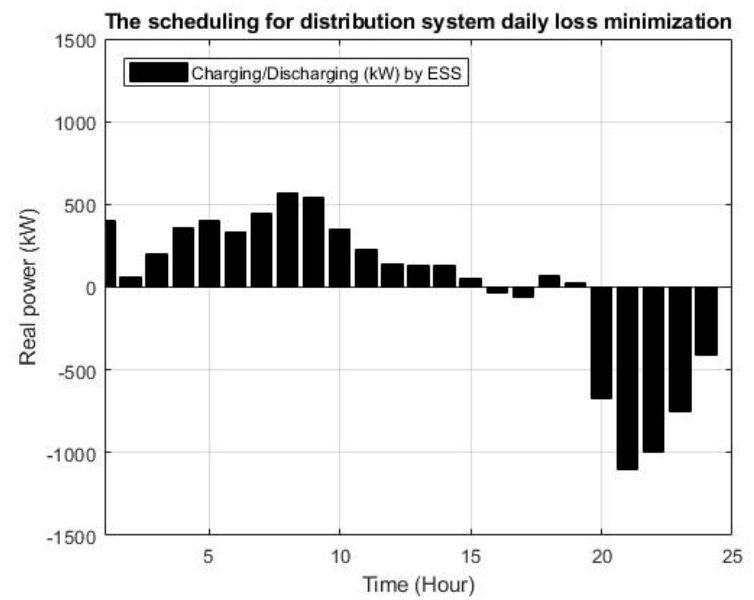

Figure. 13 The scheduling for distribution system daily loss minimization

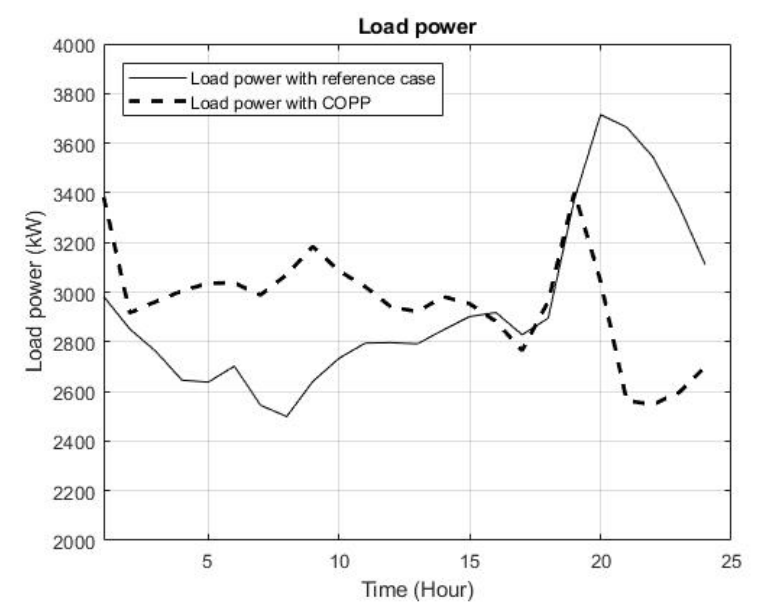

Figure. 14 The comparison of power load with and without optimal placement ESS and CBs

With the proposed MIPSO based. Fig. 11. shows that bus number 6 was chosen to install $1.5 \mathrm{MW} / 10$ MWh of ESS and buses numbers 13, 24, and 30 were placed by CBs, with the sizes of 350,600 , and 1050 KVar, respectively. 
Table 2. Hourly loss of IEEE 33-bus radial distribution test system for cases 1-4

\begin{tabular}{|c|c|c|c|c|}
\hline Time & $\begin{array}{c}\text { Case 1 } \\
\text { losses } \\
(\mathrm{kW})\end{array}$ & $\begin{array}{c}\text { Case } 2 \\
\text { lourly } \\
(\mathrm{kW})\end{array}$ & $\begin{array}{c}\text { Case } 3 \\
\text { lourly } \\
(\mathrm{kW})\end{array}$ & $\begin{array}{c}\text { Case } 4 \\
\text { losses } \\
(\mathrm{kW})\end{array}$ \\
\hline 1 & 131.7409 & 156.1866 & 87.3708 & 110.6394 \\
\hline 2 & 119.7343 & 126.0988 & 79.5606 & 83.7840 \\
\hline 3 & 111.8022 & 125.7058 & 74.3875 & 85.4428 \\
\hline 4 & 102.1437 & 125.5076 & 68.0735 & 87.8815 \\
\hline 5 & 101.4991 & 125.5794 & 67.6515 & 88.1850 \\
\hline 6 & 106.8218 & 124.8462 & 71.1337 & 86.4586 \\
\hline 7 & 94.1166 & 126.1528 & 62.8132 & 90.7851 \\
\hline 8 & 90.5947 & 127.0256 & 60.5014 & 91.7468 \\
\hline 9 & 101.7402 & 124.4514 & 67.8094 & 87.4914 \\
\hline 10 & 109.4596 & 121.0148 & 72.8576 & 84.8111 \\
\hline 11 & 114.7226 & 121.4062 & 76.2933 & 83.6642 \\
\hline 12 & 114.8860 & 121.1142 & 76.4000 & 83.5383 \\
\hline 13 & 114.4751 & 120.9067 & 76.1319 & 83.0816 \\
\hline 14 & 119.4523 & 121.2097 & 79.3769 & 81.8394 \\
\hline 15 & 124.2406 & 121.5404 & 82.4947 & 81.0080 \\
\hline 16 & 125.7144 & 121.1590 & 83.4536 & 80.3963 \\
\hline 17 & 117.6576 & 120.1695 & 78.2073 & 81.7191 \\
\hline 18 & 123.6554 & 120.8485 & 82.1139 & 80.4152 \\
\hline 19 & 171.3835 & 131.7973 & 112.9943 & 77.5112 \\
\hline 20 & 210.9875 & 144.9729 & 138.3603 & 79.3673 \\
\hline 21 & 204.7166 & 142.5138 & 134.3585 & 78.8940 \\
\hline 22 & 190.6568 & 137.9043 & 125.3664 & 77.6928 \\
\hline 23 & 168.5288 & 130.8732 & 111.1571 & 77.0083 \\
\hline 24 & 144.0933 & 124.5576 & 95.3812 & 77.4656 \\
\hline$A A L$ & $\mathbf{1 2 9 . 7 8}$ & $\mathbf{1 2 7 . 6 5}$ & $\mathbf{8 6 . 0 1}$ & $\mathbf{8 4 . 2 0}$ \\
\hline & & & & \\
\hline
\end{tabular}

Table 2 illustrated $A A L$ of the modified IEEE 33bus radial distribution test system with ESS and CBs is reduced to $84.20 \mathrm{kWh}$, lower than those case 1-3. In addition, the proposed method has been solved by GA for comparison with the PSO method. The results shown that the PSO based COPP was able to provide more efficient findings than GA when considering minimizing total loss, as shown in Table 3.

From Fig. 12, the convergence of $A A L$ is 2000 iterations. The scheduling of ESS in Fig. 13, shows that state of charge of this case is similar to case 2 . Similarly, the peak power load with ESS and CBs was decease at 21.00 o'clock shown as Fig. 14.

In this case, the curve of hourly loss was leveled smoother than ESS in case 2, as shown in Fig. 15. Meanwhile, Fig. 16. shows the voltage profiles for case 1-4. The results show that when ESS and CBs are placed optimally, significant improvements in bus voltage can be achieved when compared to other cases. Finally, the proposed method is efficiency minimize the $A A L$ by the optimal placement ESS and $\mathrm{CBs}$, considering the optimal scheduling ESS.

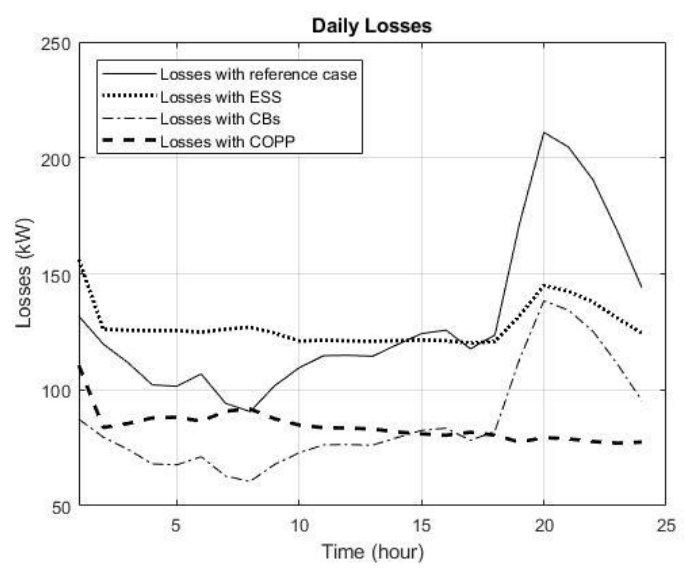

Figure. 15 The comparison of daily losses

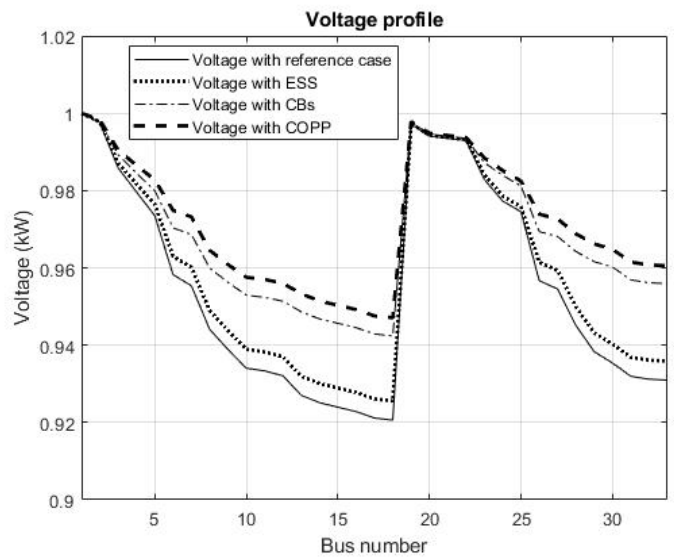

Figure. 16 The comparison of voltage profile (average from 24 hour)

Table 3. Simulation results with IEEE 33-bus radial distribution test system for cases 1-4

\begin{tabular}{|c|c|c|c|c|c|}
\hline Case & 1 & 2 & 3 & \multicolumn{2}{|c|}{4} \\
\hline Solver & - & MIPSO & MIPSO & GA & MIPSO \\
\hline OPESS(NO.) & - & 6 & - & 6 & 6 \\
\hline OPCB(NO.) & - & - & $13,24,30$ & $13,24,30$ & $13,24,30$ \\
\hline$A A L(k W h)$ & 129.78 & 127.64 & 86.01 & 84.82 & 84.20 \\
\hline MaximumV(p.u.) & 1.0000 & 1.0000 & 1.0000 & 1.0000 & 1.0000 \\
\hline MinimumV(p.u.) & 0.9206 & 0.9256 & 0.9423 & 0.9511 & 0.9470 \\
\hline$A A L$ Saving (\%) & $\mathbf{0 . 0 0}$ & $\mathbf{1 . 6 5}$ & $\mathbf{3 3 . 7 3}$ & $\mathbf{3 4 . 6 4}$ & $\mathbf{3 5 . 1 2}$ \\
\hline
\end{tabular}

\section{Conclusion}

In this paper, the COPP with DSALM sub problem is proposed, using MIPSO. The proposed method was evaluated using a modified IEEE 33-bus radial distribution test system with the load profile of the Thai power system during the summer season. When properly allocated by MIPSO, CBs can better compensate for reactive power. Meanwhile, the bus with the ESS installed can effectively determine the power consumption schedule with the PSO. The results show that, the proposed method is efficient and dependable for coordinated allocation ESS and CB in minimizing system losses, which $A A L$ saving of $35.12 \%$ when compare to the based case. 


\section{Conflicts of Interest}

The authors declare no conflict of interest.

\section{Author Contributions}

Conceptualization, K. Kaiyawong and K. Chayakulkheeree; methodology, K. Kaiyawong and K. Chayakulkheeree; software, K. Kaiyawong; validation, K. Kaiyawong and K. Chayakulkheeree; formal analysis, K. Kaiyawong and K. Chayakulkheeree; investigation, K. Kaiyawong and K. Chayakulkheeree; resources, K. Kaiyawong; data curation, K. Kaiyawong; writing - original draft preparation, K. Kaiyawong; writing-review and editing, K. Chayakulkheeree; visualization, K. Kaiyawong; supervision, K. Chayakulkheeree;.

\section{Reference}

[1] F. M. F. Flaih, L. Xiangning, S. M. Dawood, and M. A. Mohammed, "Distribution System Reconfiguration for Power Loss Minimization and Voltage Profile Improvement Using Modified Particle Swarm Optimization", In: Proc. of IEEE PES Asia-Pacific Power and Energy Conference, pp. 120-124, 2016.

[2] M. Ismail, E. Hassane, E. M. Hassan, and L. Tijani, "Power Losses Minimization in Distribution System Using Soft Open Point", In: Proc. of IEEE $1^{\text {st }}$ International Conference on Innovation Research in Applied Science, Engineering and Technology, 2020.

[3] S. Angalaeswari and K. Jamuna, "Optimal Placement and Sizing of Real Power Supporting DG in Radial Distribution Networks", In: Proc. of IEEE 2015 International WIE Conference on Electrical and Computer Engineering, pp. 342345, 2019.

[4] B. Gaur, R. Ucheniya, and A. Saraswat, "Real Power Transmission Loss Minimization and Bus Voltage Improvement Using STATCOM", In: Proc. of IEEE $20193^{\text {rd }}$ International Conference on Recent Developments in Control, pp. 236241, 2019.

[5] F. R. C. Soldevilla and F. A. C. Huerta, "Minimization of Losses in Power Systems by Reactive Power Dispatch using Particle Swarm Optimization", In: Proc. of IEEE International Universities Power Engineering Conference, 2019.

[6] D. Lukman, "Loss Minimization in Load Flow Simulation in Power System", In: Proc. of 4th IEEE International Conference on Power Electronics and Drive Systems. IEEE PEDSIndonesia. Proceedings, pp. 84-88, 2001.
[7] F. G. Bagriyanik and M. Bagriyanik, "Power Loss Minimization Using Fuzzy Multi-objective Formulation and Genetic Algorithm", In: Proc. of IEEE Bologna Power Tech Conference Proceedings, 2003.

[8] M. Azis, Hartono, and Y. Muharni, "Optimal Capacitor Placement For IEEE 118 Bus System By Using Genetic Algorithm”, In: Proc. of 2nd International Conference on High Voltage Engineering and Power Systems, 2019.

[9] M. Mahdavian, M. H. Kafi, A. Movahedi, and M. Janghorbani, "Improve Performance in Electrical Power Distribution System by Optimal Capacitor Placement Using Genetic Algorithm", In: Proc. of 14th International Conference on Electrical Engineering/Electronics/Computer/

Telecomunications and Information Technology, pp. 749-752, 2017.

[10] O. Ivanov, "Capacitor Banks Placement Optimization Improvement Using the Sperm Whale Algorithm", In: Proc. of ECAI 2019International Conference-1 $1^{\text {th }} \quad$ Edition Electronics, Computers and Artificial Intelligence, 2019.

[11] O. Ceylar and S. Paudyal, "Optimal Capacitor Placement and Sizing Considering Load Profile Variations Using Moth-Flame Optimization Algorithm", In: Proc. of International Conference on Modern Power Systems, 2017.

[12] S. JoyalIsac, K. S. Kumar, and P. V. Kumar, "Optimal Capacitor Placement in Radial Distribution System to minimize the loss using Fuzzy Logic Control", In: Proc. of International Conference on Smart Structure and System, pp. 33-40, 2013.

[13] V. Cholapandian, T. Yuvaraj, and K. Devabalaji, "Optimal Allocation of Capacitor Banks in Distribution Networks using Cuckoo Search Algorithm", In: Proc. of 7th International Conference on Electrical Energy Systems, pp. 475-478, 2021.

[14] P. Diaz, M. P. Cisneros, E. Cuevas, O. Camarena, F. Fausto, and A. Gonzalez, "A Swarm Approach for Improving Voltage Profiles and Reduce Power on Electrical Distribution Network", IEEE Access, pp. 4949849512, 2018.

[15] D. Sattianadan, M. Sudhkaran, K. Vijayakumar, and S. Vidyasagar, "Optimal Placement of Capacitor in Radial Distribution System Using PSO", In: Proc. of Chennai and Dr.MGR University Second International Conference on Sustainable Energy and Intelligent System, pp. 326-331, 2011. 
[16] R. N. D. C. Filho, "Optimal capacitor placement in radial distribution system using QPSO", Simposio Brasileiro de Sistemas Eletricos, 2018.

[17] A. Mujezinović, N. Turković, N. Dautbašić, M. M. Dedović, and I. Turković, "Use of Integer Genetic Algorithm for Optimal Allocation and Sizing of the Shunt Capacitor Banks in the Radial Distribution Networks", 18th International Symposium INFOTEHJAHORINA, 2019.

[18] G. Upadhyay, R. Saxena, and G. Joshi, "Optimal Capacitor Placement and Sizing in Distribution System Using Hybrid Approach of PSO-GA", In: Proc. of IEEE International Conference on Advance in Electrical Technology for Green Energy, 2017.

[19] M. B. Nappu, A. Arief, and M. I. Bachtiar, "Strategic Placement of Capacitor and DG for Voltage Improvement after Large Penetration of Renewable Energy Power Plant: An Indonesian Study", In: Proc. of $7^{\text {th }}$ International Conference on Renewable Energy Research and Applications, pp. 627-631, 2018.

[20] G. J. Cho, Y. S. Oh, M. S. Kim, B. Mather, and B. M. Hodge, "Optimal Capacitor Bank Capacity and Placement in Distribution Systems with High Distributed Solar Power Penetration", IEEE Power \& Energy Society General Meeting, 2017.

[21] S. B. Karanki, D. Xu, B. Venkatesh, and B. N. Singh, "Optimal Location of Battery Energy Storage Systems in Power Distribution Network for Integrating Renewable Energy Sources", IEEE Energy Conversion Congress and Exposition, pp. 4553-4558, 2013.

[22] B. C. Neagu, M. Gavrilas, R. D. Pentiuc, and E. Hopulele, "Optimal Placement of Energy Storage Systems in Microgrids Using a PSO based Approach", IEEE PES Innovative Smart Grid Technologies Europe, 2019.

[23] D. A. Rapris, P. S. Periandros, P. A. Gkaidatzis, A. S. Bouhouras, and D. P. Labridis, "Optimal Siting of BESS in Distribution Networks under High PV Penetration", In: Proc. of 53rd International Universities Power Engineering Conference, 2018.

[24] A. Alzahrani, H. Alharthi, and M. Khalid, "Minimization of Power Losses through Optimal Battery Placement in a Distributed Network with High Penetration of Photovoltaics", Energies Journal, 13, 140, 2020.

[25] A. Alzahrani, H. Alharthi, and M. Khalid, "Optimal Battery Energy Storage Placement in Highly PV-Penetreated Distribution Networks", In: Proc. of IEEE Power \& Energy Society
Innovative Smart Grid Technologies Conference, 2020.

[26] M. Nick and M. Hohmann, "Optimal Location and Sizing of Distributed Storage Systems in Active Distribution Networks", In: Proc. of IEEE Grenoble Conference, 2013.

[27] O. Babacan, W. Torre, and J. Kleissl, "Siting and sizing of distributed energy storage to mitigate voltage impact by solar PV in distribution systems", Solar Energy, 146, pp. 199-208, 2017.

[28] A. Garces, C. A. Correa, and R. Bolarios, "Optimal Operation of Distributed Energy Storage Units for Minimizing Energy Losses", In: Proc. of IEEE PES Transmission \& Distribution Conference and Exposition - Latin America, 2014.

[29] T. Khalili, A. Jafari, and E. Babaei, "Scheduling and Siting of Storages Considering Power Peak Shaving and Loss Reduction by Exchange Market Algorithm", In: Proc. of Smart Grid Conference, 2017.

[30] Y. Y. Hong, M. Y. Wu, and S. H. Lee, "Optimal Day-ahead Energy Scheduling of Battery in Distribution Systems Considering Uncertainty", In: Proc. of 5th International Conference on Green Technology and Sustainable Development, 2020.

[31] W. Lee, J. Jung, and M. Lee, "Development of 24-hour Optimal Scheduling Algorithm for Energy Storage System using Load Forecasting and Renewable Energy Forecasting", IEEE Power \& Energy Society General Meeting, 2017.

[32] Y. Oka and A. Yokoyama, "Optimal Operation Scheduling and Economical Evaluation Method of Battery Energy Storage System in Power System with a Large Penetration of Photovoltaic Generation", IEEE Grenoble Conference, 2013.

[33] J. Kennedy and R. Eberhart, "Particle Swarm Optimization", In: Proc. of IEEE Proceedings of ICNN'95 - International Conference on Neural Networks, pp. 1942-1948, 1995.

[34] K. Kaiyawong and K. Chayakulkheeree, "Optimal Energy Storage System Scheduling for Distribution System Daily Loss Minimization Using Particle Swarm Optimization”, In: Proc. of the 43rd Electrical Engineering Conference, 2020.

[35] P. Lohray, S. Gali, S. Rangisetti, and T. Nikoubin, "Rounding Technique Analysis for Power-Area \& Energy Efficient Approximate Multiplier Design", In: Proc. of IEEE 9th Annual Computing and Communication Workshop and Conference, pp. 420-425, 2019. 